\title{
Garantizando el acceso a la información pública en México: Propuesta para tratamiento de la información del portal de la Secretaria de Salud Federal
}

\author{
Por José Alfredo Hernández Landeros
}

Escuela Nacional de Biblioteconomía y Archivonomía, México

\section{Resumen}

Reflexiona sobre la necesidad de garantizar el acceso a la información a toda persona como base fundamental de una sociedad de la información. Presenta el caso de México y sus políticas públicas de información desde el Instituto Federal de Acceso a la Información. Plantea una propuesta para adoptar un sistema de gestión de información documental para la Secretaría de Salud Federal de México, basado en normas y estándares internacionales con el propósito de controlar, organizar y recuperar la información dentro de su sitio web.

\section{Palabras clave}

Acceso a la información pública ; Sociedad de la información ; Gestión de información ; Políticas públicas de información ; Dublin Core ; Instituto Federal de Acceso a la Información y Protección de Datos ; IFAI ; Secretaria de Salud Federal ; México

\section{Title}

Ensuring access to public information in Mexico: Proposal for treatment of the information portal of the Ministry of Federal Health

\begin{abstract}
Reflect on the need to ensure access to information to everyone as the foundation of an information society. The case of Mexico and its public policy information from the Federal Institute of Access to Information. A proposal to adopt an information management system documentation for the Federal Ministry of Health of Mexico, based on international norms and standards in order to control, organize and retrieve information within your site.
\end{abstract}

\section{Keywords}

Access to public information ; Information society ; Information management ; Public policy information; Dublin Core ; Federal Institute of Access to Information and Data Protection ; IFAI ; Federal Ministry of Health ; Mexico

Recibido - Received: 2011-03-30

Aceptado - Accepted: 2011-03-31 


\section{Introducción}

Durante el desarrollo de la historia del hombre, éste ha enfrentado una serie de experiencias de prueba y error, que lo han dotado de conocimientos, teorías y de tecnologías para condicionar su vivir diario. Hoy en día todas las sociedades del mundo, unas más que otras vivimos en un proceso económico, tecnológico, social y cultural a gran escala, compartido por una creciente comunicación e información a nivel globalizado.

"El mañana llega, cada vez, con más premura. El cambio es más acelerado, profundo y generalizado en todos los cambios del ser humano. Estamos viviendo la mayor transformación del mundo en la historia. Todos los sistemas: el político, el religioso, el familiar, el educativo y el organizacional están en permanente mutación. El cambio nos ha transportado a una nueva era de la información, de la tecnología y del conocimiento".

Sin duda alguna, la evolución social del hombre ha mutado desde los primeros pasos de civilización rastreados por los historiadores, antropólogos y especialistas.

A partir de 1950 se inició una nueva historia, una nueva era: la de la información, de la tecnología y del conocimiento. En esa década, en los países desarrollados, la mayoría de la gente dejó de trabajar en la manufactura y pasó a manejar información, datos estadísticos y conocimientos especializados. Pasó a trabajar en el campo de servicios, de la supervisión de personal, de gerencia y liderazgo.

En esta era la mayoría de los seres humanos dejó de usar sus manos y sus músculos como herramienta principal para la sobrevivencia. La fuerza física dejo de ser, para la mayoría, la generadora de riqueza y de la obtención de la remuneración económica. Hoy por hoy, la mayoría de las personas utilizan principalmente sus capacidades de pensar y de relación, la educación escolar, técnica o universitaria, la capacitación y actualización permanente como fuente de supervivencia, competitividad y seguridad para el futuro. Las computadoras y los medios electrónicos de comunicación están acelerando este cambio.

La era de la información es un término aplicado al período en el cual el movimiento de información se volvió más rápido que el movimiento físico, esta era de la información y del conocimiento está generando una nueva forma de vida en todos los sistemas sociales, políticos y económicos. Transformando la cultura de los pueblos y de las organizaciones.

La corriente paradigmática de la era de la información alude a quebrantar la brecha digital, donde todo ser humano tiene las mismas oportunidades y derechos al libre acceso de la información, para beneficio propio y desarrollo personal, bajo la salvedad de la legalidad y protección intelectual de quien emana dicha información. Así como en la consulta transparente de las funciones sobre los ejercicios gubernamentales como evidencia de rendición de cuentas para la ciudadanía. Es por ello que los gobiernos, y sectores públicos, deben promover y adoptar valores que garanticen la igualdad y la equidad en el acceso a la información. Además de ofrecer las mismas oportunidades de desarrollo económico y social a todo ser humano. 


\section{México en la sociedad de información.}

En esta dirección, México en 2006 publica en el Diario Oficial de la Federación, la Ley General para la Igualdad entre Mujeres y Hombres. El cual decreta en su "artículo 1.Regular y garantizar la igualdad entre mujeres y hombres y proponer los lineamientos y mecanismos institucionales que orienten a la Nación hacia el cumplimiento de la igualdad sustantiva en los ámbitos público y privado."ii

Asimismo en esta ley podemos observar el valor de la equidad en el art. 3, que de igual manera también la equidad es mencionada en el artículo 123 constitucional, en el apartado "a", que en términos generales menciona: que las normas de trabajo tienden a conseguir el equilibrio y la justicia social en las relaciones entre trabajadores y patrones, que el trabajo es un derecho y un deber social, que no es un artículo de comercio, que exige respeto para las libertades y dignidad de quien lo presta, y debe efectuarse en condiciones que aseguren la vida, la salud y un nivel decoroso para el trabajador y su familia, y que no podrán establecerse distinciones entre los trabajadores por motivos de raza, sexo, edad, credo, religión, doctrina política o condición social, y que es de interés social promover y vigilar la capacitación y el adiestramiento de los trabajadores.

En cuestiones de acceso a la información y de igualdades equitativas para que el mexicano cuente con los mismos conocimientos y condiciones para manejar las Tecnologías de Información y de Comunicación. La ley General para la Igualdad entre Mujeres y Hombres, empieza a enunciar el deber federal y municipal, de promover la educación en todos los aspectos socioeconómicos y culturales, los cuales podemos notar en los siguientes artículos:iii

- Capitulo cuatro; Art. 16.- De conformidad con lo dispuesto en la presente Ley y las leyes locales de la materia, corresponde a los Municipios: V. Fomentar la participación social, política y ciudadana dirigida a lograr la igualdad entre mujeres y hombres, tanto en las áreas urbanas como en las rurales.

- Art. 17.- La Política Nacional en Materia de Igualdad entre mujeres y hombres deberá establecer las acciones conducentes a lograr la igualdad sustantiva en el ámbito, económico, político, social y cultural.

- Capitulo quinto; Art. 39: V.- Reforzar la cooperación y los intercambios de información sobre los derechos humanos e igualdad entre hombres y mujeres con organizaciones no gubernamentales y organizaciones internacionales de cooperación para el desarrollo.

\section{México y las políticas públicas de información.}

“Las políticas públicas son las acciones del gobierno que actúan directamente sobre las necesidades sociales y están encaminadas a influir sobre la vida de los ciudadanos. Se originan a partir de un proceso de decisiones que se realizan a lo largo de un tiempo determinado bajo una secuencia racional de administración y gestión pública, dándole así el carácter de público al ser generadas dentro del marco de procedimientos, instituciones y organizaciones gubernamentales, entre ellas del poder legislativo." iv $Y$ están atendidas a través del análisis científico y la negociación colectiva, propia de un 
entorno democrático.

Las políticas públicas de información son fundamentales para garantizar el acceso y el derecho a la información. Sin embargo "el cierre de la brecha digital de acceso plantea un desafío importante en materia de políticas públicas por su carácter dinámico, que obliga a un constante ajuste de sus objetivos, en la medida que surgen nuevas tecnologías."v

En México las políticas públicas de información están presentes en nuestra constitución en los siguientes artículos:vi

- Art. 6: señala que podemos expresar libremente nuestras ideas siempre y cuando no se ataque a la moral, no se perjudiquen a terceros y no se cometa ningún delito ni se perturbe el orden público.

- Art. 7: nos habla de la libertad de escribir y publicar sobre cualquier materia, garantizando la libertad de prensa y la libertad de imprenta, manteniendo las reservas de respeto y seguridad.

- Art. 8: referente a la obligación de los funcionarios públicos de respetar el derecho de petición de información por parte de los ciudadanos, siempre y cuando que se haga por escrito y de una forma pacífica y respetuosa. El funcionario tiene la obligación de entregar la información solicitada a la brevedad posible.

Asimismo otras leyes en México que aluden a esto son:

- Ley de imprenta.(1917)

- La ley general de bibliotecas.(1987)

- Ley de información estadística y geografía.(1980)

- Ley del Diario oficial de la Federación y Gacetas gubernamentales.(1986)

- Ley Federal de derechos de autor.(1996)

- Ley de fomento para la lectura y el libro.(2000)

- Ley y ciencia de la tecnología.(2002)

- Ley de la transparencia y acceso a la información pública gubernamental.(2002)

\section{El Instituto Federal de Acceso a la Información y Protección de Datos.}

Con mayor importancia, México a partir del 2002, ha avanzado enormemente en la rendición de cuentas del gasto presupuestario de los Poderes de la Unión, así como en las atribuciones y funciones que desempeñan los funcionarios públicos, asiendo pública la información, y otorgándole libre acceso a ella, a todo ciudadano de nuestro país que así lo demande y queda plasmado por la Ley Federal de Transparencia y Acceso a la información y por medio del Instituto Federal de Acceso a la Información y Protección de Datos, siendo éste ultimo una institución al servicio de la sociedad y es el encargado de:

1) Garantizar el derecho de acceso a la información pública gubernamental.

2) Proteger los datos personales que están en manos del gobierno federal. 
3) $\mathrm{Y}$ resolver sobre las negativas de acceso a información que las dependencias o entidades del gobierno federal hayan formulado.

\section{Teniendo como marco normativo:}

“El derecho de acceso a la información favorece la transparencia en el gobierno y la rendición de cuentas de todos los servidores públicos, lo cual mejorará la eficiencia de las instituciones federales y la calidad de sus servicios. A partir del 12 de junio del 2003, la Ley Federal de Transparencia y Acceso a la Información Pública Gubernamental obliga a todas las dependencias y entidades del gobierno federal a dar acceso a la información contenida en sus documentos, respecto, entre otras cosas, a su forma de trabajo, al uso de los recursos públicos, sus resultados y desempeño. Cualquier persona puede solicitar información a las instituciones federales y obtenerla en forma rápida y sencilla, sin necesidad de identificarse, ni justificar el uso que dará a la misma. Además, esta Ley garantiza el derecho de las personas a la vida privada, al obligar a las instituciones a proteger los datos personales que tienen en sus archivos o bases de datos. De esta forma, distingue la información gubernamental, que es pública, de la información sobre las personas, que es confidencial. La Ley, aprobada en junio del año 2002, es producto de la participación de grupos de la sociedad que llevaron una iniciativa propia del Ejecutivo Federal al Congreso y los legisladores, quienes la aprobaron en forma unánime. Con base en la Ley, fue creado el Instituto Federal de Acceso a la Información y Protección de Datos (IFAI), un organismo autónomo encargado de garantizar a todas las personas el acceso a la información pública y la protección de sus datos personales que posee el gobierno federal."vii

"Con esta ley no sólo se establece la obligación de las dependencias y entidades gubernamentales de hacer pública la información que poseen. También establece la obligación de transparencia que cada dependencia y entidad debe observar tomando en cuenta las restricciones y acepciones marcadas por la ley, con el fin de salvaguardar la seguridad nacional y el orden público así como la protección a la privacidad personal"viii. El IFAI impulsa los principios y los valores de actuación de los servidores públicos y de la sociedad en el combate a la corrupción.

A partir de la entrada en vigor de la Ley Federal de Transparencia y Acceso a la Información Pública Gubernamental, más de 250 dependencias y entidades del gobierno federal tienen la obligación de atender las solicitudes de información de la ciudadanía.

\subsection{Portales de transparencia y acceso a la información pública en México: El caso del portal de la Secretaria de Salud Federal.}

La Secretaria de Salud Federal a través de su portal conforme lo estipulado a la Ley Federal de Transparencia y Acceso a la Información Pública Gubernamental y el Instituto Federal de Acceso a la Información y Protección de Datos (IFAI), pone a disposición toda información derivada de sus documentos, trabajos y obligaciones al acceso abierto a toda persona que así lo solicite. Buscando "consolidar una política de Estado que permita fortalecer las buenas prácticas dentro de la Administración Pública. Esto se hará con un enfoque de gestión más honesto, transparente y con una renovada vocación de rendición de cuentas."ix 
Sin embargo la organización de los documentos disponibles para la transparencia y rendición de cuentas dentro del portal de la Secretaría de Salud, se da por medio de listados localizados en el menú de "Transparencia" del propio sitio. Así mismo el sitio, cuenta con un metabuscador por palabras claves, que es un motor de búsqueda de palabra por palabra dentro de los textos digitales, almacenados en el servidor.

Subjetivamente, éste sistema podría ser eficiente para usuarios familiarizados con la localización de información dentro del portal. Sin embargo en la medida que aumenta la información, los desplegados de listas resultan cada vez más difíciles de consultar y las búsquedas son más costosas en tiempos y menos manejables, a pesar de contar con un metabuscador de palabra por palabra, éste no resulta suficiente porque de la misma manera los desplegados de listas de los documentos electrónicos, podrían ser enormes ó en el peor caso, obtener documentos intranscendentes con las necesidades informativas.

No obstante la mayor dificultad se encuentra en la propia organización y control de la información por parte de su generador.

\subsection{Propuesta para el tratamiento de la información del portal de la Secretaria de Salud Federal.}

\subsubsection{Propuesta}

Se plantea que el portal de la Secretaria de Salud Federal adopte un sistema de base de datos con aplicación cliente servidor, que no es más que una arquitectura que consiste básicamente en un cliente que realiza peticiones a otro programa (servidor) que le da respuesta.

Ésta base de datos debe permitir administrar, organizar y controlar documentos electrónicos, dando respuestas a peticiones condicionadas por medio de campos descriptivos previamente catalogados; ya sea por campos únicos, metadatos predefinidos, plantillas y formatos. Con el fin de permitir a los ciudadanos buscar registros a través de metadatos, texto completo, búsquedas truncadas y con operadores boléanos.

"Cualquiera que pretenda encontrar información en línea utilizando uno de los servicios de búsqueda Web utilizados hoy en día, probablemente habrá experimentado la frustración al encontrar cientos, o miles, de ocurrencias con la posibilidad ilimitada de refinar o de hacer una búsqueda más precisa.

La adopción a gran escala de estándares y prácticas descriptivas para los recursos electrónicos mejorará la recuperación de recursos relevantes en cualquier contexto donde la recuperación es crítica." $\mathrm{x}$

\subsubsection{Uso de estándares internacionales para el tratamiento de información}

Por lo anterior una base de datos de recursos electrónicos debe de apoyarse de estándares internacionales para reducir los riesgos de una mala planeación y al mismo tiempo contar con la posibilidad de intercambiar registros documentales con sistemas afines.

Una sabia inversión a largo plazo es desarrollar un sistema de arquitectura abierta, y 
estructura modular que soporte estándares industriales como: Dublin Core, MARC 21, FRBR, Unicode, ODBC, OpenURL, HTML, XML y protocolos de intercambio de información; HTTP, Z39.50 y el protocolo OAI (Open Archives Initiative) para recolectar metadatos. A continuación algunos ejemplos de estos estándares:

\section{El Estándar Dublin Core}

Es un estándar de metadatos que presenta una serie de elementos para describir una amplia gama de recursos electrónicos, que permite tener sistemas de recuperación más inteligentes y sus mayores beneficios son:xi

- Desarrollar estándares de metadatos para la recuperación de información en Internet a través de distintos dominios.

- Definir el marco para la interoperabilidad entre conjuntos de metadatos.

- Facilitar el desarrollo de conjuntos de metadatos específicos de una disciplina o comunidad que trabaja dentro del marco de la recuperación de información.

De los cuales figuran los siguientes campos descriptivos:xii

\section{a) Elementos del contenido}

- Título (Title). Se refiere al título que lleva por nombre el documento.

- Materia (Subject). En este campo se hace referencia a los diversos temas que puede contener el material.

- Descripción (Description). En este campo se hace un breve resumen sobre el contenido del objeto digital.

- Fuente (Source). Es como una pequeña ficha bibliográfica que se elabora para asentar los datos sobre la procedencia del documento original.

- Lenguaje (Language). En este campo se establecen las siglas correspondientes al idioma en que se presenta la publicación.

- Relación (Relation). Este campo tiene que ver con el material principal u objetos de su misma referencia, ya sea una colección, una serie, un documento, etc.

- Cobertura (Coverage). Este campo se refiere al proyecto o sitio donde estará resguardada la información. Aquí pueden anotarse fechas, zonas geográficas.

b) Elementos de propiedad intelectual

- Autor (Creator). Aquí se anota el autor intelectual de la obra o documento original.

- Editor (Publisher). Este campo se refiere al sitio o colección responsable, a la que está adscrito el material.

- Colaborador (Contributor). En este campo se anotan, si es que se da el caso, el nombre u organización que contribuyó a la creación del material, que no se especificó en la parte de Autor. 
- Derechos (Rights). Se anota en este campo el nombre o la institución a la cual pertenece el material y lo facilitó.

\section{c) Elementos de aplicación}

- Fecha (Date). Se anota la fecha de elaboración del registro.

- Tipo (Type). Aquí se menciona la presentación que tiene el objeto digital, ya sea como texto, audio, video, etcétera.

- Formato (Format). En este campo se registra el tipo de extensión con que se presenta el objeto digital, ya sea HTML, JPG, GIFF o PDF.

- Identificador (Identifier). Se refiere a la dirección electrónica de origen a la que está adscrito el material. Para ello se utilizan las siglas URL.

\section{El formato MARC 21}

Es un modelo de registros bibliográficos legibles por maquina, es decir; una computadora puede leer e interpretar los datos contenidos en un registro catalográficos. A la vez que es un modelo normalizado de intercambio, desarrollado por la Biblioteca del Congreso de Washington, E.A.U.

"El formato MARC es universalmente aceptado por los centros de información, porque los softwares de automatización de los centros de información proporcionan diversas facilidades para la creación de los registros y porque con su adopción, es posible realizar la catalogación mediante la copia de registros elaborados por otros centros, con lo que se reduce enormemente el esfuerzo necesario para la actualización del catálogo."xiii

MARC 21 contiene un gran número de campos descriptivos que se adecuan a cada documento para crear puntos de acceso que permitan su recuperación. El llenado de los campos es normalizado y estandarizado por el uso regalas de catalogación, esquemas de clasificación y lenguajes controlados.

Las etiquetas de uso más frecuentes son:xiv

\begin{tabular}{|c|l|}
\hline Etiqueta & \multicolumn{1}{c|}{ Función } \\
\hline 010 & que marca al Número de Control de la Biblioteca del Congreso (LCCN) \\
\hline 020 & que marca al Número Internacional Normalizado para Libros(ISBN) \\
\hline 100 & que marca al asiento principal bajo nombre personal (autor) \\
\hline 245 & $\begin{array}{l}\text { que marca a la información del título (incluido el título propiamente } \\
\text { dicho, otra información sobre el título, y la mención de responsabilidad) }\end{array}$ \\
\hline 250 & que marca a la mención de edición \\
\hline 260 & que marca a la información sobre la publicación \\
\hline 300 & que marca a la descripción física \\
\hline 440 & que marca al asiento secundario de serie \\
\hline 520 & que marca a la nota de sumario o comentario \\
\hline 650 & que marca al encabezamiento temático de materia \\
\hline 700 & $\begin{array}{l}\text { que marca al asiento secundario bajo nombre personal(coautor, editor o } \\
\text { ilustrador) }\end{array}$ \\
\hline
\end{tabular}


Para accesar a la información de un recurso electrónico, hay que agregar la etiqueta 865 .El campo puede ser utilizado en un registro bibliográfico de un recurso cuando el subconjunto de recursos o de que esté disponible por vía electrónica. "Además, puede ser utilizada para localizar y acceder a una versión electrónica de un recurso no electrónico descrito en el registro bibliográfico o un recurso electrónico relacionado." $x \mathrm{x}$

Es decir; éste campo permite redireccionar el registro a una FTP, URL o HTTP u otros protocolos y direcciones vía red.

\subsubsection{Dinámica de actualización y presentación de la información.}

Asimismo el portal de la Secretaria de Salud Federal, debe revisar constantemente su contenido informativo con el fin de actualizar el sitio, con las últimas noticias en el ámbito de la salud en México.

Es pertinente que ésta información siempre sea tratada en términos periodísticos para que tengan un contexto significativo para los ciudadanos. Sin entrar a detalles la información puede ser presentada en uno de los siguientes textos:

- Texto expositivo-explicativo "cuyo texto tiene por objetivo principal el expresar información o ideas con la intención de mostrar y de explicar o hacer más comprensible dichas informaciones."xvi

- Discurso o texto argumentativo entendido "como el conjunto de estrategias de un orador que se dirige a un auditorio con vistas a modificar el juicio de dicho auditorio, conseguir su adhesión o hacer que admitan una determinada situación, idea, etc.", xvii es decir, el texto argumentativo pretende persuadir las emociones o el intelecto de las personas para tomar un postura determinada. La persuasión emocional, consiste en hacer analogías de sucesos de la vida común, mientras que la persuasión intelectual hace hincapié a la argumentación de citas bibliográficas o estadísticas.

Por último la información debe ser presentada secundariamente como un: Mensaje publicitario, que tiene por "estrategia primaria del lenguaje publicitario de intentar captar por un momento la atención del lector mediante una imagen atractiva o mediante un mensaje especial. Desde el punto de vista lingüístico, en la publicidad contemporánea para conseguir la necesaria connotación que requiere la persuasión, se emplean recursos fónicos, morfosintácticos, léxicos y retóricos para hacer mas deseables los productos que se ofrecen."xviii 


\section{Bibliografía}

Álvarez Angulo, Teodoro. (2000). Textos expositivos-explicativos y argumentativos. España: Octaedro. p. 91

Bermello Crespo, Luis. (2004). Procedimiento para la catalogación por copia de revistas en base de datos a texto completo en Internet. [en línea]. Cuba. [Consulta: 26 febrero 2011]. Disponible en: <http://eprints.rclis.org/bitstream/10760/5909/1/aci3604.pdf>

Biblioteca Del Congreso. (2003). ¿Qué es un registro MARC, y parqué es importante?. [en línea]. [Consulta: 2 Marzo 2011]. Disponible en:

<http://www.loc.gov/marc/umbspa/um01a06.html>

Diccionario de la Real Academia de la Lengua Española. (2010). [en línea]. Madrid.

[Consulta: 27 febrero 2011] disponible en:

<http:/ / buscon.rae.es/draeI/SrvltConsulta?TIPO_BUS=3\&LEMA=igualdad>

Dublin Core Metadata Initative. Usando el Dublin Core. (2003.) [en línea]. [Consulta: 26 febrero 2011]. Disponible en: <http://www.sedic.es/usando_dublin_core.pdf $>$

Guerra, Massiel, Jordán, Valeria. (2010). Políticas públicas de la información en América Latina ¿una misma visión?. Santiago de Chile: Comisión económica para América Latina y el Caribe. 26 p.

Instituto Federal de Acceso a la Información y Protección de Datos. (2010). [en línea]. México: [Consulta: 26 febrero 2011]. Disponible en:

<http://www.ifai.org.mx/Acercalfai/Marco $>$

Ley general para la igualdad entre mujeres y hombres. (2006). [en línea]. México: Cámara de Diputados del Congreso de la Unión. [Consulta: 26 febrero 2011].

Disponible en: <http://www.diputados.gob.mx/LeyesBiblio/pdf/LGIMH.pdf>

Marc Standards. 856- electronic location and access. (2003). [en línea]. Washington: Library of Congress [Consulta: 2 Marzo 2011]. Disponible en:

<http://www.loc.gov/marc/bibliographic/bd856.html>

Metadatos y acceso a la recuperación de la información: metadatos para describir e identificar un documento en la red. (2010). [en línea]. [Consulta: 26 febrero 2011]. Disponible en: <http://www.metadatos-xmlrdf.com/metadatos/dublin-core>

Salazar Vázquez, Francisco Fabián. (2008). La ley federal de transparencia y acceso a la información pública gubernamental en el marco del derecho a la información. México: UNAM. 42 p.

Secretaría De Salud Federal. Trasparencia y rendición de cuentas. (2008). [en línea]. México. [Consulta: 2 Marzo 2011]. Disponible en: <http://portal.salud.gob.mx/contenidos/ptrc/ptrc2008-2012.html>

Siliceo A., Alfonso. (1999). Liderazgo, valores y cultura organizacional. México: McGraw-Hill. 220 p. 


\section{Datos del autor}

\section{José Alfredo Hernández Landeros}

Egresado de la Escuela Nacional de Biblioteconomía y Archivonomía de México. Auditor técnico en Biblioteconomía en ISO 9001:2008, Det Norske Veritas México. Se desempeña como catalogador y clasificador en Library Outsourcing Service. alfreherland@hotmail.com

\footnotetext{
' SILICEO A., Alfonso. Liderazgo, valores y cultura organizacional. México: McGraw-Hill. 1999. p. 3

ii Ley general para la igualdad entre mujeres y hombres. [en línea]. México: Cámara de Diputados del Congreso de la Unión, 2006 [Consulta: 26 febrero 2011]. Disponible en: http://www.diputados.gob.mx/LeyesBiblio/pdf/LGIMH.pdf

iii Ibíd. p. 2-13

iv SALAZAR VÁZQUEZ, Francisco Fabián. La ley federal de transparencia y acceso a la información pública gubernamental en el marco del derecho a la información. México: UNAM, 2008. p. 14

${ }^{\vee}$ GUERRA, Massiel, JORDÁN, Valeria. Políticas públicas de la información en América Latica ¿una misma visión?. Santiago de Chile: Comisión económica para América Latina y el Caribe. p. 11

vi SALAZAR VÁZQUEZ, Francisco. Op. Cit. 14-15

vii INSTITUTO FEDERALDE ACCESO A LA INFORMACIÓN Y PROTECCION DE DATOS. [en línea]. México: [Consulta: 26 febrero 2011]. Disponible en: http://www.ifai.org.mx/Acercalfai/Marco

viii SALAZAR VÁZQUEZ. Op. cit. p. 23

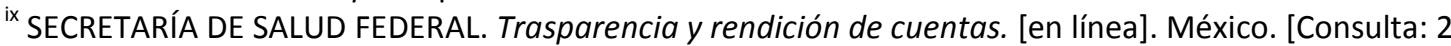
Marzo 2011]. Disponible en: http://portal.salud.gob.mx/contenidos/ptrc/ptrc2008-2012.html

${ }^{x}$ DUBLIN CORE METADATA INITATIVE. Usando el Dublin Core. [en línea]. [Consulta: 26 febrero 2011]. Disponible en: http://www.sedic.es/usando_dublin_core.pdf

${ }^{x i}$ Metadatos y acceso a la recuperación de la información: metadatos para describir e identificar un documento en la red [en línea]. [Consulta: 26 febrero 2011]. Disponible en: http://www.metadatos-xmlrdf.com/metadatos/dublin-core

xii Ibíd.

xiii BERMELLO CRESPO, Luis. Procedimiento para la catalogación por copia de revistas en base de datos a texto completo en Internet. [en línea]. Cuba. [Consulta: 26 febrero 2011]. Disponible en: http://eprints.rclis.org/bitstream/10760/5909/1/aci3604.pdf

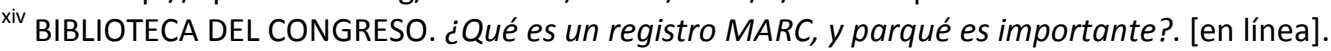
[Consulta: 2 Marzo 2011]. Disponible en: http://www.loc.gov/marc/umbspa/um01a06.html

${ }^{\mathrm{xv}}$ MARC STANDARDS. 856- electronic location and access. [en línea]. Washington: Library of Congress [Consulta: 2 Marzo 2011]. Disponible en: http://www.loc.gov/marc/bibliographic/bd856.html

xvi ÁLVAREZ ANGULO, Teodoro. Textos expositivos-explicativos y argumentativos. España: Octaedro, 2000 p. 16

xvii Ibíd. p.48

xviii Ibíd. p. 58
} 